\title{
Mouse Strains
}

National Cancer Institute

\section{Source}

National Cancer Institute. Mouse Strains. NCI Thesaurus. Code C14420.

Breeds of mice utilized for biological research. 